\title{
Epidemiological update on the dengue situation in the Western Pacific Region, 2012
}

\author{
Yuzo Arima, ${ }^{a}$ May Chiew ${ }^{a}$ and Tamano Matsuia on behalf of the Emerging Disease Surveillance and \\ Response Team, Division of Health Security and Emergencies, World Health Organization Regional Office \\ for the Western Pacific \\ Correspondence to the Emerging Disease Surveillance and Response Unit (e-mail: outbreak@wpro.who.int).
}

Dengue has caused a substantial public health burden in the Western Pacific Region. To assess this burden and regional trends, data were collated and summarized from indicator-based surveillance systems on dengue cases and deaths from countries and areas in the Western Pacific Region. In 2012, dengue notifications continued to increase with 356838 dengue cases reported in the Region (relative to 244855 cases reported in 2011) of which 1248 died. In the Asia subregion, the notification rate was highest in Cambodia, the Philippines and the Lao People's Democratic Republic (316.2, 198.9 and 162.4 per 100000 population, respectively), and in the Pacific island countries and areas, the notification rate was highest in Niue, the Marshall Islands and the Federated States of Micronesia (8556.0, 337.0 and 265.1 per 100000 population, respectively). All four serotypes were circulating in the Region in 2012 with considerable variabilitiy in distribution. Regional surveillance provides important information to enhance situational awareness, conduct risk assessments and improve preparedness activities.

$\mathrm{n}$ recent years, dengue has become a major public health concern in the Western Pacific Region, resulting in substantial morbidity, mortality and economic cost. $^{1-3}$ Such public health and economic burdens have become clear not only from national surveillance data but also from operational research studies aimed at estimating the dengue disease burden. ${ }^{4}$ The epidemiology and virology of dengue continues to display complex behaviour with serotype interactions, antibodydependent enhancement and cross-immunity, climate and notable gender and age distributions. ${ }^{5-8}$ Notifications of dengue cases - most likely an underestimate of the true burden ${ }^{4,9}$ - have increased over the past decade, with more than 200000 annual cases consistently reported in the Western Pacific Region since 2007,1,2 and nearly 250000 dengue cases reported in the Region in $2011^{2}$

This analysis reports the 2012 annual regional dengue surveillance data collated by the World Health Organization (WHO) Western Pacific Regional Office.

\section{METHODS}

Regional dengue data for 2012 were collated from indicator-based surveillance systems from countries and areas in the Region. Data were either sent to WHO from the ministries of health or collected from their websites.

Additional data were provided from Australia, Cambodia, the Lao People's Democratic Republic, Malaysia, the Philippines, Singapore and Viet Nam. A summary of the dengue surveillance systems, case definitions, laboratory sampling methods and serotype data are included. Malaysia and the Philippines were the only countries with changes since the 2011 annual report. $^{2}$ For Malaysia, all cases fulfilling the clinical criteria for dengue or those with a positive laboratory confirmation were reported; for the first time, NS1 antigen detection was included as a testing method. In the Philippines, the 2009 dengue case classification system continued to be rolled out in 2012 following training at the regional, provincial and municipal/city

\footnotetext{
Emerging Disease Surveillance and Response, Division of Health Security and Emergencies, World Health Organization Regional Office for the Western Pacific, Manila, Philippines. 
Figure 1. Number of reported dengue cases and case fatality rates in the Western Pacific Region, 2000 to 2012*

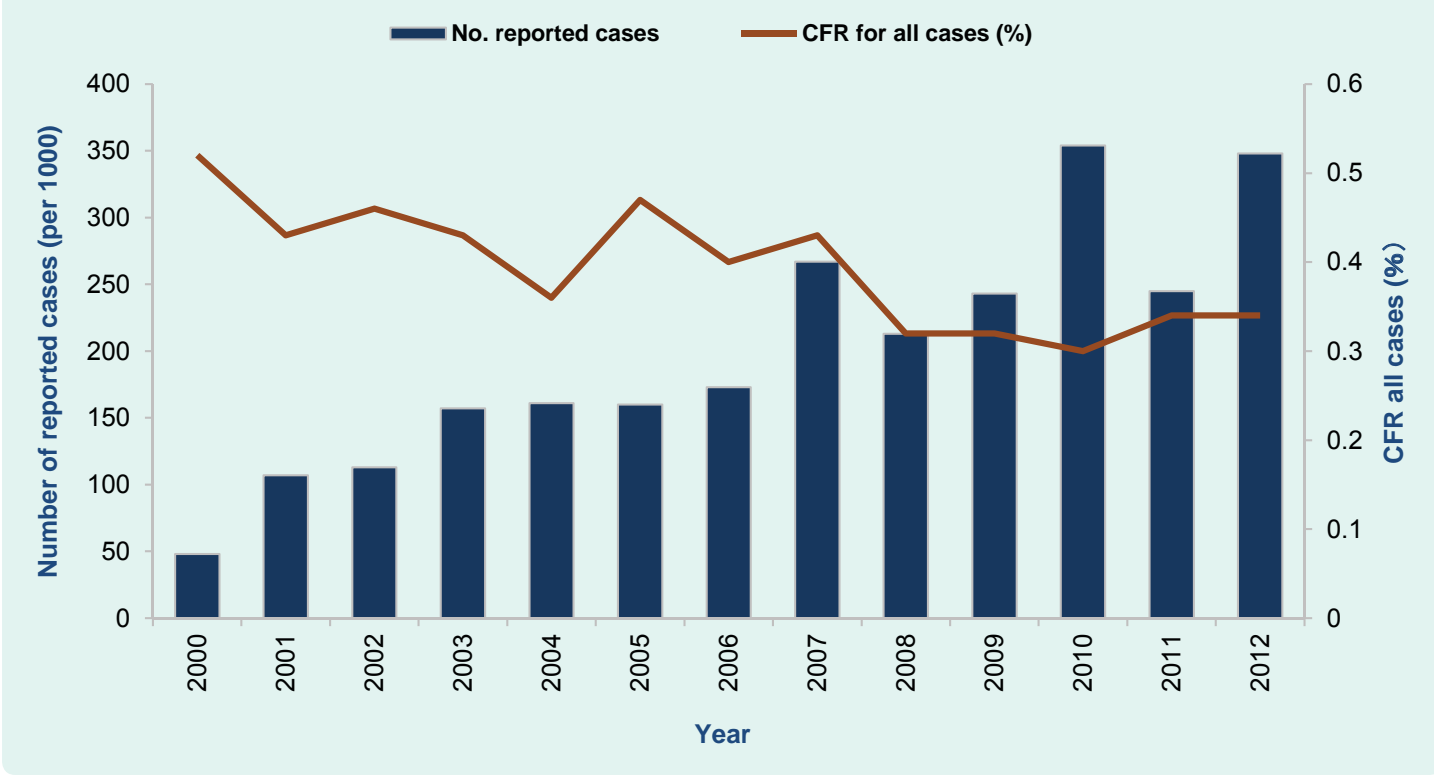

Source: World Health Organization Regional Office for the Western Pacific.

* Dengue surveillance and reporting systems vary by country.

CFR, case fatality rate.

health offices. Training was ongoing at the end of 2012, and hence the new case definition was not applied nationwide by the year end.

\section{RESULTS}

\section{Dengue in the Western Pacific Region}

In 2012, Western Pacific Region Member States reported a total of 356838 dengue cases of which 1248 died for a case fatality rate of $0.34 \%$ (Figure 1 ). In the Asia subregion, both the notification rate and the absolute number of reported dengue cases were highest in the Philippines (Table 1). In the Pacific subregion, there were large increases in notification rates in Niue, Fiji and New Caledonia relative to $2011 .^{2}$ While Australia reported more than 1500 laboratory-confirmed cases (Table 2), the majority were imported cases.

While laboratory sampling schemes and confirmation methods vary by country, most of the countries in this report were using the updated (2009) dengue case classification system in 2012 (Table 3).

For those countries providing additional data, all except for Singapore reported a higher number of cases in 2012 compared with 2011 (Table 2). Overall, the number of cases reported in 2012 was more than $40 \%$ higher than in $2011 .^{2}$

\section{Asia subregion}

\section{Cambodia}

In 2012, Cambodia reported 42362 clinical dengue cases (189 fatal), considerably more than the 15980 cases reported in the previous year (Table 3 ). Notifications peaked in week 27 ( $n=2447$ cases) in July (Figure 2), similar to 2011 (peak in July) and 2010 (peak in August). Among those aged more than 15 years, there was a higher proportion of males (male-to-female ratio: 1.2 to 1 ). Among the 500 laboratory-tested cases, 463 (93\%) were confirmed. Three serotypes circulated with the predominant serotype being DEN-1 (DEN-1 $n=368$ [98\%], DEN-2 $n=5$ [1\%] and DEN-4 $n=3$ $[1 \%])$.

\section{The Lao People's Democratic Republic}

In 2012, 9952 clinical dengue cases (22 fatal) were reported, more than double that of 2011 (Table 2). Notifications peaked in week $40(n=555$ cases $)$ in October (Figure 2), later than in 2011 (peak in September) and 2010 (peak in August). Among the 871 


\begin{tabular}{|c|c|c|c|c|c|}
\hline Countries/territories $^{\dagger}$ & Cases & $\begin{array}{l}\text { Notification } \\
\text { per } 100000\end{array}$ & Deaths & $\begin{array}{c}\text { Case fatality } \\
\text { rate }(\%)\end{array}$ & $\begin{array}{c}\text { Population } \\
\text { (in thousands) }\end{array}$ \\
\hline \multicolumn{6}{|l|}{ Asia subregion } \\
\hline Brunei Darussalam & 290 & 71.43 & 0 & 0.00 & 406 \\
\hline Cambodia & 42362 & 316.23 & 189 & 0.45 & 13396 \\
\hline China & 575 & 0.04 & 0 & 0.00 & 1370537 \\
\hline China, Hong Kong Special Administrative Region & 53 & 0.75 & 0 & 0.00 & 7068 \\
\hline China, Macao Special Administrative Region & 24 & 4.35 & & 0.00 & 552 \\
\hline Japan & 220 & 0.17 & 0 & 0.00 & 128056 \\
\hline Republic of Korea & 145 & 0.30 & & 0.00 & 48875 \\
\hline Lao People's Democratic Republic & 9952 & 162.40 & 22 & 0.22 & 6128 \\
\hline Malaysia & 21900 & 77.52 & 35 & 0.16 & 28251 \\
\hline Mongolia & 0 & 0.00 & 0 & - & 2780 \\
\hline Philippines & 187031 & 198.94 & 921 & 0.49 & 94013 \\
\hline Singapore & 4632 & 89.35 & 2 & 0.04 & 5184 \\
\hline Viet Nam & 86026 & 100.00 & 79 & 0.09 & 86025 \\
\hline Total for subregion & 353210 & 19.72 & 1248 & 0.35 & 1791271 \\
\hline \multicolumn{6}{|l|}{ Pacific subregion } \\
\hline Australia & 1542 & 6.90 & 0 & 0 & 22342 \\
\hline Fiji & 705 & 82.55 & 0 & 0 & 854 \\
\hline Kiribati & 243 & 240.59 & 0 & 0 & 101 \\
\hline Marshall Islands & 182 & 337.04 & 0 & 0 & 54 \\
\hline Micronesia (Federated States of) & 273 & 265.05 & 0 & 0 & 103 \\
\hline New Caledonia & 478 & 194.31 & 0 & 0 & 246 \\
\hline New Zealand & 77 & 1.86 & 0 & 0 & 4143 \\
\hline Niue & 128 & 8556.00 & 0 & 0 & 1.5 \\
\hline Total for subregion & 3628 & 13.03 & 0 & 0 & 27845 \\
\hline TOTAL & 356838 & 19.62 & 1248 & 0.35 & 1819116 \\
\hline
\end{tabular}

Source:World Health Organization Regional Office for the Western Pacific

* Dengue surveillance and reporting systems vary by country.

The following countries and territories did not report dengue data: American Samoa, Cook Islands, French Polynesia, Guam, Nauru, the Commonwealth of Northern Mariana Islands, Palau, Papua New Guinea, Pitcairn Islands, Samoa, Solomon Islands, Tokelau, Tonga, Tuvalu, Vanuatu and Wallis and Futuna.

laboratory-tested cases, 449 (52\%) were confirmed. While all four serotypes circulated, the predominant serotype was DEN-3 (DEN-3 $n=164[80 \%]$, DEN-1 $n=23$ [11\%], DEN-2 $n=18$ [9\%] and DEN-4 $n=1$ $[<1 \%])$.

\section{Malaysia}

In 2012, Malaysia reported 21900 cases (35 fatal), similar to 2011 and relatively low compared to years before 2011 (Table 3). However, this was the first year that those with a laboratory confirmation, regardless of clinical manifestation, were included (Table 3 ). The highest number of cases $(n=602)$ was reported during week 8 in February; higher notifications were observed from December through February (Figure 2), similar to 2011 when a peak was observed in January. Among the 7797 laboratory-tested cases, 6506 (83\%) were confirmed. All four serotypes were detected with an almost equal distribution (DEN-3 $n=263$ [31\%], DEN-1 $n=222$ [26\%], DEN-4 $n=185$ [22\%] and DEN-2 $n=184[22 \%])$.

\section{The Philippines}

In 2012, the Philippines reported 187031 clinical cases (921 fatal), a 48\% increase compared with 2011 (Table 2), with a peak in the month of August ( $n=31$ 999) (Figure 2). Those aged $5-14$ years were the age group with the largest number of cases. Among the 
Table 2. Reported number of dengue cases, deaths and case fatality rates from Cambodia, the Lao People's Democratic Republic, Malaysia, the Philippines, Singapore, Viet Nam and Australia, 2008-2012*

\begin{tabular}{|c|c|c|c|c|c|c|c|c|c|c|c|c|c|c|c|}
\hline \multirow{2}{*}{ Countries } & \multicolumn{3}{|c|}{2008} & \multicolumn{3}{|c|}{2009} & \multicolumn{3}{|c|}{2010} & \multicolumn{3}{|c|}{2011} & \multicolumn{3}{|c|}{2012} \\
\hline & Cases & Deaths & CFR (\%) & Cases & Deaths & CFR (\%) & Cases & Deaths & CFR (\%) & Cases & Deaths & CFR (\%) & Cases & Deaths & ; CFR (\%) \\
\hline Cambodia & 9542 & 65 & 0.68 & 11699 & 38 & 0.32 & 12500 & 58 & 0.30 & 15980 & 73 & 0.46 & 42362 & 189 & 0.45 \\
\hline $\begin{array}{l}\text { Lao People's } \\
\text { Democratic } \\
\text { Republic }\end{array}$ & 4149 & 21 & 0.51 & 7214 & 12 & 0.17 & 22929 & 46 & 0.20 & 3905 & 7 & 0.18 & 9952 & 22 & 0.22 \\
\hline Malaysia & 49335 & 112 & 0.23 & 41486 & 88 & 0.21 & 46171 & 134 & 0.29 & 19884 & 36 & 0.18 & 21900 & 35 & 0.16 \\
\hline Philippines & 39620 & 373 & 0.94 & 57819 & 548 & 0.95 & 135355 & 793 & 0.59 & 125975 & 654 & 0.52 & 187031 & 921 & 0.49 \\
\hline Singapore & 7031 & 10 & 0.14 & 4497 & 8 & 0.18 & 5363 & 6 & 0.11 & 5330 & 6 & 0.11 & 4632 & 2 & 0.04 \\
\hline Viet Nam & 96451 & 97 & 0.10 & 105370 & 87 & 0.08 & 128831 & 55 & 0.04 & 69680 & 61 & 0.09 & 86026 & 79 & 0.09 \\
\hline Australia & 563 & 0 & 0 & 1401 & 0 & 0 & 1171 & 0 & 0 & 820 & 0 & 0 & 1542 & 0 & 0 \\
\hline Total & 206692 & 678 & 0.33 & 229486 & 781 & 0.34 & 352321 & 1070 & 0.30 & 241574 & 837 & 0.35 & 353445 & 1248 & 0.35 \\
\hline
\end{tabular}

Source: World Health Organization Regional Office for the Western Pacific

* Dengue surveillance and reporting systems vary by country.

CFR, case fatality rate.

Figure 2. Reported number of dengue cases by calendar week (Cambodia, the Lao People's Democratic Republic, Malaysia, New Caledonia and Singapore) and month (Australia and the Philippines), 2012

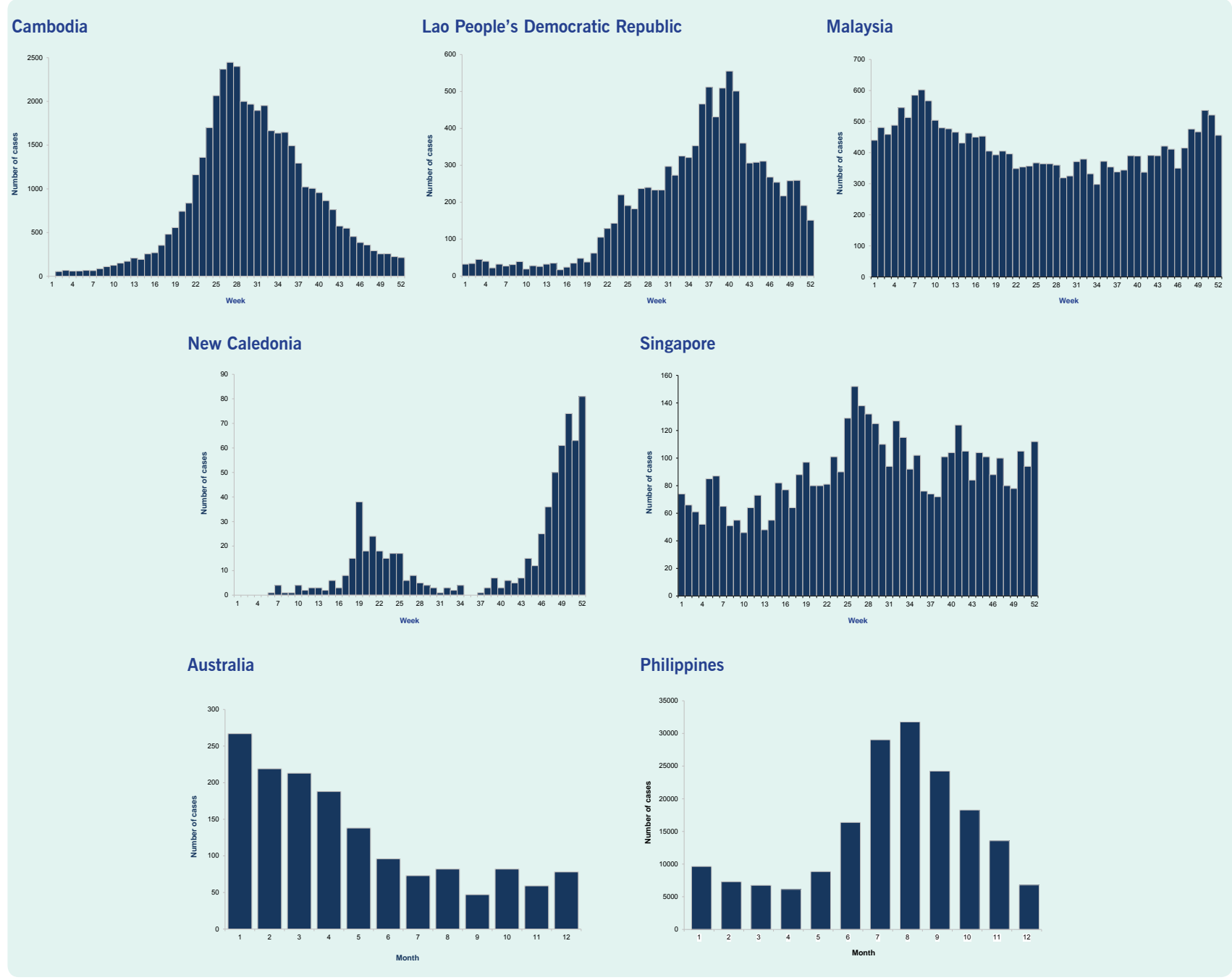


Table 3. Dengue case definitions, laboratory sampling and testing methods used for surveillance in Cambodia, the Lao People's Democratic Republic, Malaysia, the Philippines, Singapore, Viet Nam and Australia, 2012*

\begin{tabular}{|c|c|c|c|}
\hline \multirow[b]{2}{*}{ Country } & \multicolumn{2}{|c|}{ Case definition } & \multirow[b]{2}{*}{ Laboratory sampling and testing method } \\
\hline & Clinical criteria $^{+}$ & $\begin{array}{l}\text { Laboratory } \\
\text { confirmation }\end{array}$ & \\
\hline Cambodia & $\begin{array}{l}2009 \text { dengue case } \\
\text { classification }^{\dagger}\end{array}$ & No & $\begin{array}{l}\text { Five sentinel sites send maximum of } 5 \text { samples per week for } \\
\text { testing, focusing primarily on children. Confirmation is based } \\
\text { on enzyme-linked immunosorbent assay (ELISA), polymerase } \\
\text { chain reaction (PCR) and/or virus isolation. If the time interval } \\
\text { between date of onset and sample collection is within } 5 \text { days, } \\
\text { ELISA, haemagglutination inhibition assay, PCR and virus } \\
\text { isolation are performed; if this interval is }>5 \text { days, only ELISA and } \\
\text { haemagglutination inhibition assay are performed. }\end{array}$ \\
\hline $\begin{array}{l}\text { Lao People's } \\
\text { Democratic } \\
\text { Republic }\end{array}$ & $\begin{array}{l}2009 \text { dengue case } \\
\text { classification }^{\dagger}\end{array}$ & No & $\begin{array}{l}\text { A proportion of dengue cases, such as ad hoc outbreak specimens } \\
\text { and samples sent from provincial hospitals, are tested by ELISA. } \\
\text { IgM positive specimens for which the time between date of onset } \\
\text { and collection is }<5 \text { days were selected for serotyping at one } \\
\text { facility, while another serotyped all specimens received. }\end{array}$ \\
\hline Malaysia & $\begin{array}{l}\text { Fever or history of fever } \\
\text { AND } \geq 2 \text { of the following: } \\
\text { headache, retro-orbital pain, } \\
\text { myalgia, arthralgia, rash, } \\
\text { haemorrhagic manifestation, } \\
\text { leucopenia } \\
\text { OR cases with positive } \\
\text { laboratory result }\end{array}$ & No & $\begin{array}{l}\text { No sampling scheme: confirmation based on serology (IgM) or } \\
\text { antigen detection (NS1). }\end{array}$ \\
\hline Philippines & $\begin{array}{l}2009 \text { dengue case } \\
\text { classification }^{\dagger} \\
\text { Acute onset of fever } 2-7 \text { days } \\
\text { with } \geq 2 \text { of following: } \\
\text { headache, retro-orbital pain, } \\
\text { myalgia, arthralgia, rash, } \\
\text { haemorrhagic manifestations, } \\
\text { leucopenia }\end{array}$ & No & $\begin{array}{l}\text { A proportion of suspected dengue cases are tested by serology } \\
\text { (IgM) or PCR. }\end{array}$ \\
\hline Singapore & $\begin{array}{l}\text { Acute onset of fever lasting } \\
2-7 \text { days with } \geq 2 \text { of following: } \\
\text { headache, backache, } \\
\text { myalgia, rash, retro-orbital } \\
\text { pain, bleeding, leucopenia }\end{array}$ & Required & $\begin{array}{l}\text { All clinically diagnosed cases are laboratory tested and only those } \\
\text { positive by serology (IgM) or PCR/NS1 are registered. }\end{array}$ \\
\hline Viet Nam & $\begin{array}{l}2009 \text { dengue case } \\
\text { classification }^{\dagger}\end{array}$ & No & $\begin{array}{l}\text { A proportion of dengue cases are tested through serology and a } \\
\text { limited number by virus isolation. }\end{array}$ \\
\hline Australia & $\begin{array}{l}\text { Fever, headache, arthralgia, } \\
\text { myalgia, rash, nausea and } \\
\text { vomiting }\end{array}$ & Required & $\begin{array}{l}\text { All clinically diagnosed cases are laboratory tested and only those } \\
\text { confirmed by the following method are registered: isolation/detection } \\
\text { of dengue virus OR IgG seroconversion or significant increase in } \\
\text { antibody level or } \geq 4 \text {-fold rise in titre to dengue virus OR detection } \\
\text { of dengue virus-specific IgM in cerebrospinal fluid OR detection of } \\
\text { dengue virus-specific IgM in serum. }\end{array}$ \\
\hline
\end{tabular}

* Only the minimum criteria required for fulfilling a clinical dengue case definition are included here; additional signs and symptoms required for more severe forms (e.g. dengue haemorrhagic fever, dengue shock syndrome) are not listed here.

A probable dengue case is defined as any case with fever and two or more of the following: nausea, vomiting, rash, aches and pains, positive tourniquet test, leucopenia and any warning sign. A case with warning signs is defined as a clinically diagnosed case with any of the following: abdominal pain or tenderness, persistent vomiting, clinical fluid accumulation, mucosal bleed, lethargy, restlessness, liver enlargement $>2 \mathrm{~cm}$ and increase in haematocrit concurrent with rapid decrease in platelet count. Severe dengue is defined as severe plasma leakage leading to any of the following: shock, fluid accumulation with respiratory distress OR severe bleeding as evaluated by clinician OR severe organ involvement of liver (aspartate amino transferase or alanine amino transferase $\geq 1000$ ), central nervous system (impaired consciousness) or heart and other organs. 
165 laboratory-tested cases, 142 (86\%) were confirmed and among the serotyped cases, DEN-1 predominated (DEN-1 $n=128$ [90\%], DEN-2 $n=10$ [7\%] and DEN-3 $n=4[3 \%])$.

\section{Singapore}

In 2012, Singapore reported 4632 laboratory-confirmed cases of dengue (2 fatal), less than that reported in 2011 or 2010 (Table 2), with a peak in week 26 ( $n=152$ cases) in June (Figure 2 ), similar to 2011 which peaked in July. While all four serotypes were detected, the predominant serotype among 1333 serotyped cases was DEN-2 (DEN-2 $n=988$ [74\%], DEN-1 $n=258$ [19\%], DEN-3 $n=76$ [6\%] and DEN-4 $n=11[1 \%]$ ).

\section{Viet Nam}

In 2012, Viet Nam reported 86026 clinical cases (79 fatal), greater than that reported in 2011 (Table 3). Among the 13222 laboratory-tested cases, 5317 (40\%) were confirmed. All four serotypes were detected with DEN-1 most common (DEN-1 $n=319$ [32\%], DEN-2 $n=262$ [26\%], DEN-4 $n=235$ [23\%] and DEN-3 $n=188[19 \%])$.

\section{Pacific subregion}

\section{Australia}

In 2012, Australia reported 1542 laboratory-confirmed dengue cases ( 0 fatal), the largest number reported in the past five years (Table 2), with a peak in the month of January ( $n=267$ cases) (Figure 2) similar to 2011. In North Queensland, among 28 locally acquired dengue cases, the predominant serotype was DEN-1 (14 DEN-1, 7 DEN-3, 1 DEN-2 and 6 untyped); 13 of the 28 cases were male. Among 41 imported cases, 22 were DEN2, 13 were DEN-1, 3 were DEN-3, 1 was DEN-4 and 2 were not typed (personal communication, Dr Sonia Harmen, Tropical Public Health Services Cairns, Division 1 Family Health and Well-being Cairns and Hinterland Hospital and Health Service, Queensland Government).

From the Pacific subregion, Niue had the highest notification rate (8556 per 100000 population; 128 cases), Fiji reported more than 700 cases (82.6 per 100000 population) and New Caledonia had a large increase in notifications in the last quarter of 2012 (Figure 2) with nearly 500 cases (194.3 per 100000 population) reported compared with a single case in 2011 (Table 1). In New Zealand, where dengue is not endemic, 77 cases were reported in 2012 with 76 being classified as imported cases.

\section{DISCUSSION}

Dengue continued to burden the Western Pacific Region in 2012 with the overall number of notifications greater than previous years. More than 1000 cases were reported each from Australia, Cambodia, the Lao People's Democratic Republic, Malaysia, the Philippines, Singapore and Viet Nam; except for Singapore, they all reported an increase in cases compared with 2011. Seasonally, dengue notifications followed historic patterns, increasing and peaking during the wet season in Cambodia, the Lao People's Democratic Republic, the Philippines and Viet Nam. While some countries had the same serotype predominate as in the previous year (Cambodia, Malaysia, Singapore and Viet Nam), others saw a change, including Australia and the Lao People's Democratic Republic. In the latter, DEN-3 became predominant in 2012 compared with DEN-1 during $2010^{1}$ and $2011 .^{2}$

In the Pacific subregion, although large outbreaks were observed in the Federated States of Micronesia and the Marshall Islands in 2011, notifications were lower in 2012. However, Fiji and Niue experienced a high number of dengue notifications, and there was an increase in notifications observed in New Caledonia that was the beginning of the largest outbreak ever reported in the territory. ${ }^{10}$ Although dengue-specific surveillance is not conducted in Papua New Guinea, circulation of the virus there is well recognized from epidemiologic and phylogenetic analyses of imported cases in Australia and elsewhere. ${ }^{11}$

The notable changes in notification rates and shifts in serotype distribution since 2011 highlight once again the need for ongoing surveillance, information-sharing and assessment. Timely notification at the local level acts as a trigger for early response, such as vector control and outbreak investigations to interrupt transmission locally. At a country or regional level, routine and timely information-sharing enhances situational awareness and feeds into risk assessment and preparedness activities such as risk communication for neighbouring countries or those with close trade/travel links (e.g. dengue has been introduced from Asia to isolated Pacific islands ${ }^{12}$ ). 
Importantly, since routine biweekly reporting of the regional dengue situation started in 2010, information has been regularly shared through wider public health surveillance networks such as ProMED ${ }^{13}$ and translated for local use by Member States to raise awareness for travellers' health. ${ }^{14}$ Regional information-sharing activities to promote early response are in line with the WHO Asia Pacific Strategy for Emerging Diseases framework to strengthen national and regional capacities for surveillance and response.

As for previous regional analyses of dengue, ${ }^{1,2}$ there are important limitations in the surveillance data, both for interpreting the actual burden of dengue (e.g. underreporting) and trends over time (e.g. changes in disease awareness, diagnosis/testing/reporting behaviour). For example, in Malaysia, NS1 antigen positive specimens were added as an approved laboratory testing method for surveillance, and regardless of clinical manifestation, patients with laboratory confirmation were included as cases in 2012. In the Philippines, the 2009 dengue classification system continued to be rolled out to replace the clinical case definition that was still in much use during 2011. Comparisons across countries also require caution, as Australia and Singapore report laboratory-confirmed cases only, while Cambodia, the Lao People's Democratic Republic, Malaysia, the Philippines and Viet Nam primarily clinically suspected cases; such practices may lead to under- or overreporting. Importantly, case fatality rates are affected not only by clinical management but also by health-careseeking behaviour, reporting practices of clinicians, case definitions, timing of report, follow-up and verification procedures; these factors can also differ across Member States and over time. Sampling schemes for laboratory confirmation also differ across Member States and may not be systematic or representative, potentially limiting the interpretability of the reported serotype distribution. Lastly, the 2009 dengue case classification scheme became incorporated by many Member States with dengue surveillance systems during 2011 and 2012, affecting comparability with previous years.

While acknowledging these limitations, there will continue to be a need for region-wide sharing of dengue data on a routine and timely basis. Direct comparisons of notification rates and case fatality rates between countries should be avoided; within countries, however, historic and consistent seasonal trends have been observed along with potentially important changes such as serotype distribution. Such surveillance data can also be used for mathematical models ${ }^{6}$ and to provide baseline dengue surveillance data when a dengue vaccine enters the market. Lastly, in this rapidly developing and interconnected region, the ever-increasing importation of cases into non-endemic areas signifies the importance of monitoring and sharing dengue information by all countries. ${ }^{15}$

\section{Conflicts of interest}

None declared.

\section{Funding}

None.

\section{Acknowledgements}

The authors would like to thank all the WHO country fffice staff and Member States in the Western Pacific Region for assisting with the surveillance data collection and reporting.

\section{References:}

1. Arima Y, Matsui T. Epidemiologic update of dengue in the Western Pacific Region, 2010. Western Pacific Surveillance and Response Journal, 2011, 2(2):4-8. doi:10.5365/wpsar.2011.2.2.005 pmid:23908882

2. Arima $Y$ et al. Epidemiologic update on the dengue situation in the Western Pacific Region, 2011. Western Pacific Surveillance and Response Journal, 2013, 4(2):47-54. doi:10.5365/ wpsar.2012.3.4.019 pmid:24015372

3. Shepard DS, Undurraga EA, Halasa YA. Economic and disease burden of dengue in Southeast Asia. PLoS Neglected Tropical Diseases, 2013, 7:e2055. doi:10.1371/journal.pntd.0002055 pmid:23437406

4. Vong S et al. Under-recognition and reporting of dengue in Cambodia: a capture-recapture analysis of the National Dengue Surveillance System. Epidemiology and Infection, 2012, 140:491-499. doi:10.1017/S0950268811001191 pmid:21733251

5. Duong $\mathrm{V}$ et al. Complex dynamic of dengue virus serotypes 2 and 3 in Cambodia following series of climate disasters. Infection, Genetics and Evolution, 2013, 15:77-86. doi:10.1016/j. meegid.2012.05.012 pmid:22677620

6. Reich NG et al. Interactions between serotypes of dengue highlight epidemiological impact of cross-immunity. Journal of the Royal Society, Interface, 2013, 10:20130414. doi:10.1098/ rsif.2013.0414 pmid:23825116

7. Anker M, Arima Y. Male-female differences in the number of reported incident dengue fever cases in six Asian countries. Western Pacific Surveillance and Response Journal, 2011, 2(2):17-23. doi:10.5365/wpsar.2011.2.1.002 pmid:23908884

8. Prasith $\mathrm{N}$ et al. Assessment of gender distribution in dengue surveillance data, the Lao People's Democratic Republic. Western Pacific Surveillance and Response Journal, 2013, 4(2):17-24. doi:10.5365/wpsar.2012.3.4.020 pmid:24015367 
9. Undurraga EA, Halasa YA, Shepard DS. Use of expansion factors to estimate the burden of dengue in Southeast Asia: a systematic analysis. PLoS Neglected Tropical Diseases, 2013, 7:e2056. doi: 10.1371/journal.pntd.0002056 pmid:23437407

10. Dupont-Rouzeyrol M et al. Epidemiological and molecular features of dengue virus type-1 in New Caledonia, South Pacific, 20012013. Virology Journal, 2014, 11:61. doi:10.1186/1743422X-11-61 pmid:24684835

11. Warrilow D, Northill JA, Pyke AT. Sources of dengue viruses imported into Queensland, Australia, 2002-2010. Emerging Infectious Diseases, 2012, 18:1850-1857. doi:10.3201/ eid1811.120014 pmid:23092682

12. Wilder-Smith A, Gubler DJ. Geographic expansion of dengue: the impact of international travel. The Medical Clinics of
North America, 2008, 92:1377-1390, x. doi:10.1016/j. mcna.2008.07.002 pmid:19061757

13. ProMED. Published Date: 2012-09-17 16:43:48. Subject: PRO/EDR> Dengue/DHF update 2012 (43): Asia Archive Number: $20120917.1297396 \quad$ (http://www. promedmail.org/direct.php?id=20120917.1297396, accessed 26 February 2015).

14. Quarantine Information - dengue situation updates - Asia [in Japanese]. Tokyo, Office Ministry of Health, Labour and Welfare Japan, 2014.

15. Nakamura $\mathrm{N}$ et al. Incidence of dengue virus infection among Japanese travellers, 2006 to 2010. Western Pacific Surveillance and Response Journal, 2012, 3(2):39-45. doi: 10.5365/ wpsar.2011.2.3.002 pmid:23908911 